Part of Journal of Research of the National Bureau of Standards, Volume 19, July 1937

\title{
ARC AND SPARK SPECTRA OF LUTECIUM
}

\author{
By William F. Meggers and Bourdon F. Scribner
}

\section{ABSTRACT}

A new description of conventional arc and spark spectra of lutecium (cassiopeium) in the wave-length range 2000 to $11000 \mathrm{~A}$ has been completed. Wavelength measurements and intensity estimates are presented for 650 lines, 25 of which represent band heads presumably due to $\mathrm{LuO}, 250$ are characteristic of neutral Lu atoms (Lu I spectrum), 370 of singly ionized atoms (Lu II spectrum), and the remainder probably belong to doubly ionized atoms (Lu III spectrum). These data should aid in the spectroscopic identification and analysis of lutecium and serve as a basis for further study of spectral structures, hyperfine structures, and Zeeman effects.

CONTENTS

Page

I. Introduction

II. Experiments

III. Results

IV. References

\section{INTRODUCTION}

The chemical elements with atomic numbers 58 to 71 , inclusive, known as the rare-earth group, are characterized by nearly identical chemical properties which prevent their complete separation or purification by chemical means [1]. ${ }^{1}$ Slight differences in physical properties permit partial separation from each other (by fractional crystallization or by electrolysis), but it is doubtful if any rare-earth element of spectroscopic purity has ever been available for study. Impure rare-earth materials, or misidentification of spectra, account for the mistaken discovery of new elements, such as demonium, by Rowland; incognitium and ionium, by Crookes; celtium, by Urbain; denebium, dubhium, eurosamarium, and welsium, by Eder [2].

The atomic-emission spectra of these elements are characterized, in general, by great complexity and considerable variability depending on the type of excitation. The complexity (aside from impurities) is due in part to large families of spectral terms, usually of high multiplicity [3], and in part to relatively low ionizing potentials [4] which result in the excitation of several successive atomic spectra in addition to rather complicated molecular spectra. The most extensive published descriptions of these spectra were made more than a quarter of a century ago by Exner and Haschek [5], and a tabulation of the number of lines they observed in arc and spark spectra gives some idea of the relative complexity of the spectra of rare-earth elements. See table 1 .

1 Numbers in brackets refer to literature citations at the end of this paper. 
TABLE 1.-Number of lines published for rare-earth spectra by Exner and Haschek [5]

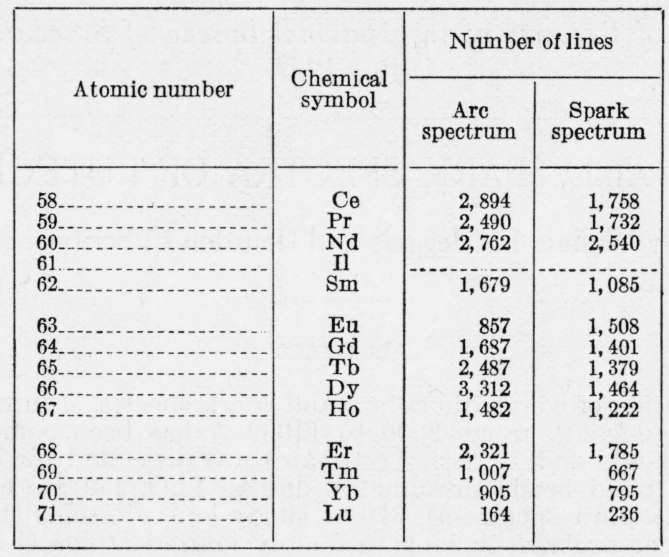

The past 15 years has been an epoch-making period for the development of the quantum theory and for the analysis of complex spectra, but the rare-earth group was the last to succumb to analysis. Taking advantage of the relative simplicity of Lu spectra, and guided by comparisons with theoretically similar spectra, the present authors succeeded in finding the first regularities [6], which were published in 1930. It was first necessary, however, to make new descriptions of the spectra since the older data were incomplete and unreliable. The purpose of the present paper is to make public, for purposes of spectroscopic identification, our complete description of the conventional arc and spark spectra of lutecium.

The first data published concerning Lu spectra consisted of 33 wave lengths (2701.8 to 3647.9 A) by Urbain [7] and 62 lines (2603.38 to $6222.6 \mathrm{~A}$ ) by Auer von Welsbach [8], both of whom discovered the element practically simultaneously, the former naming it Jutecium $(\mathrm{Lu})$ and the latter cassiopeium (Cp). A little later Exner and Haschek [5] gave values for 164 arc lines (2392.26 to $6945.30 \mathrm{~A}$ ) and 236 spark lines (2195.64 to $6463.38 \mathrm{~A}$ ). Some information concerning lines characteristic of neutral and of ionized atoms may be gleaned from a comparison of these arc and spark tables, but the separation by means of these data is often false or ambiguous. In 1910 Eder and Valenta [9] published 48 lines (5476.94 to 7126.23) observed in the arc spectrum, and in 1911 gave reproductions of spectrograms [10], a table of 116 wave lengths for the arc spectrum (2390.84 to $7126.23 \mathrm{~A}$ ) and 79 wave lengths for the spark spectrum (2603.38 to $6463.31 \mathrm{~A}$ ). In 1915 Eder [11], having obtained a purer sample from Auer, published a new description of the arc spectrum listing 263 lines (2397.17 to $7237.89 \mathrm{~A}$ ), but many of these are not now identifiable with Lu. Considering the briefness of published lists, the poor quality of early observations, the limited range of spectra, and the impure materials used, it must be admitted that the description of Lu spectra was in a very unsatisfactory state, and the necessity for making new observations before analysis of spectral structure could succeed is apparent. Our preliminary report on regularities in Lu spectra gave improved data for 100 classified lines [6]. A temperature classification of Lu spectra by King [12] lists 102 lines showing perfect agreement 
with our separation of lines according to excitation stages. Incidentally, it may be added that the close coincidence of a strong $\mathrm{Lu}$ I line with the almost omnipresent Ca II line, 3968.47 A, noted by King, was mentioned 20 years earlier by Eder and Valenta [10.]

\section{EXPERIMENTS}

Our first measurements of $\mathrm{Lu}$ spectra were made in 1929 with a sample of lutecium oxide obtained from Eder 10 years earlier. It was part of his collection of rare earths prepared by Auer. This sample contained a considerable amount of $\mathrm{Yb}$ and some $\mathrm{Tm}$, so that it was not possible to complete the description of $\mathrm{Lu}$ spectra without also investigating those of $\mathrm{Yb}$ and $\mathrm{Tm}$. The Eder collection just referred to contained a sample of ytterbium oxide but lacked any specimen of $\mathrm{Tm}$ salt. The $\mathrm{Yb}$ was found to be contaminated with both $\mathrm{Lu}$ and $\mathrm{Tm}$, so the identification of $\mathrm{Lu}$ and $\mathrm{Yb}$ lines could be effected, but considerable uncertainty remained about the removal of all Tm impurity lines from either $\mathrm{Lu}$ or $\mathrm{Yb}$. When this situation was explained to Professor B. S. Hopkins, he kindly presented new samples of Lu, Yb, and Tm oxides prepared at the University of Illinois. With these materials we were able to check and extend our former lists of $\mathrm{Lu}$ and $\mathrm{Yb}$ lines, and also to make greatly improved descriptions of Tm spectra. This work was facilitated by the comparative purity of the Illinois samples. Although the Lu spectrograms show many $\mathrm{Yb}$ and some Tm lines, and the Tm spectrograms show both $\mathrm{Yb}$ and $\mathrm{Lu}$, the best $\mathrm{Yb}$ sample is practically free of both $\mathrm{Lu}$ and $\mathrm{Tm}$.

In order to insure the correct assignment of spectral lines to their origin, both as regards chemical element and stage of ionization, two procedures were followed: (1) The spectra of $\mathrm{Lu}, \mathrm{Yb}$, and $\mathrm{Tm}$ (and $\mathrm{Er}$ ) were photographed side by side so that each line could be ascribed to the proper element on the basis of intensities, and (2) are and spark spectra of each element were photographed in juxtaposition, so that intensity comparisons would separate lines from different excitation stages. In each case the rare-earth salt was fused on pure silver or copper electrodes for the production of arc spectra, and the identical silver electrodes were used in making spark spectrograms. Each pair of spectrograms was flanked by iron spectra for wave-length measurements, and the latter by blank spectra of the silver or copper. The arcs were operated with direct current of 5 amperes and applied potential of 220 volts, while the sparks were generated by high voltage $(10,000$ to 40,000 ) discharges from mica condensers of 0.006-microfarad capacity connected parallel with the spark in the secondary circuit of the transformers.

Ultraviolet spectrograms from 2000 to $3000 \mathrm{~A}$ were made with a Hilger $E_{1}$ quartz spectrograph. The interval 2500 to $8500 \mathrm{~A}$ was photographed with concave-grating spectrographs having either 15,000 or 20,000 lines per inch, while 7500 to $11000 \mathrm{~A}$ was studied with a similar grating spectrograph having 7,500 lines per inch. The dispersion values for the first-order spectra of the gratings are 5.0, 3.7, and $10 \mathrm{~A} / \mathrm{mm}$, respectively. Since only a fraction of a gram of each salt was used for making all the required spectrograms, it was imperative to get the maximum efficiency out of the spectrographs.

Hilger Schumann plates were used for the shortest waves, Eastman 33 plates covered the region 2300 to $4800 \mathrm{~A}$, and sensitized plates ( $E K$ types $F, N, Q$ ) recorded the longer waves [13]. 


\section{RESULTS}

All wave-length measurements were made relative to iron standards (except 2000 to $2200 \mathrm{~A}$, which was measured relative to copper standards), the values being those adopted by the International Astronomical Union [14], or those published by Burns and Walters [15]. Every line listed in table 2 was observed on two or more spectrograms (except a small number with questioned intensities observed only on strongest exposures). The stronger $\mathrm{Lu}$ lines were measured on 5 to 10 spectrograms, and many of them were measured an equal number of times on $\mathrm{Yb}$ and $\mathrm{Tm}$ spectrograms, where they appeared as impurities. The concordance of values from different spectrograms and the coincidence of wave lengths with impurity lines indicates that the final results for most lines are correct within $0.01 \mathrm{~A}$, but errors of $0.02 \mathrm{~A}$ or more may exist among faint lines or those showing wide hyperfine structure. In some cases, it was difficult to decide if close lines represented fine or hyperfine structure, but our intention was to give only the center of gravity of complex lines. This hyperfine structure of Lu lines, first mentioned by the authors [6], was further commented on by King [12], and then studied in some detail by Schüler and Schmidt [16], and by Gollnow [17]. A nuclear moment of $5 / 2$ was first announced [18] for $175 \mathrm{Lu}$, but this was later [16] increased to 7/2. Gollnow [17] reported the discovery of a new Lu isotope (mass number 173 or 177) whose relative abundance is estimated at 1.5 percent, and whose spin is $7 / 2$ or possibly $9 / 2$. Although the hyperfine structure of Lu lines is not resolved in our spectrograms, it widens some of the line images to $1 \mathrm{~A}$ or more, and may thus result in some uncertainty as to the centers of gravity and perhaps leads to overestimated intensities of wide lines. Some notes about $h f s$ are given at the end of table 2 .

Wave-length measurements and estimated relative intensities for 650 lines characteristic of $\mathrm{Lu}$ atoms are presented in table 2. About 250 of these lines belong to neutral atoms, 370 to singly ionized atoms, and 5 or 6 to doubly ionized atoms. In addition, some 25 band heads presumably due to $\mathrm{LuO}$ are given. Since spark spectra were not observed above $6800 \mathrm{~A}$, the assignment of longer waves to I and II spectra is based principally on the term analysis of these spectra, and may possibly require some slight corrections when these analyses are completed. It is not unlikely that a few real Lu lines may have been discarded on account of coincidence with impurity lines, but the greatest deficiency in this description is probably in Lu spark lines masked by silver lines, or by air lines, and unobserved above $6800 \mathrm{~A}$, unless they appear in the arc spectrum. The number of classified lines in $\mathrm{Lu}$ I and $\mathrm{Lu}$ II spectra has been increased to 200, but details of these analyses will be postponed until more complete and until Zeeman effects required for the interpretation of the spectral terms have been observed.

The strongest line characteristic of neutral Lu atoms is $6004.52 \mathrm{~A}$, or possibly $4518.57 \mathrm{~A}$, while the strongest line characteristic of singly ionized atoms is $5476.69 \mathrm{~A}$ with $6221.87 \mathrm{~A}$ and $6463.12 \mathrm{~A}$ next. It may be expected that the most intense lines will also be the most persistent or sensitive for detecting minute traces of $\mathrm{Lu}$, so any sources in which neutral atoms predominate (flames, low-voltage arcs) should be examined for $6004.52 \mathrm{~A}$, but if ionized atoms are most abundant 
5476.69 A will reveal the lowest concentration of Lu. It appears that on account of the easy ionization of $\mathrm{Lu}$ atoms, the strongest Lu II lines have greater intensity than any Lu I lines in the ordinary 220 -volt arc with short gap, and 5476.69 and $6221.87 \mathrm{~A}$ may be regarded as the most persistent lines of conventional are and spark sources.

Attention is called to the fact that 19 of the 24 lines published by Urbain [19] as characteristic of "celtium" are now identified as lutecium.

TABLE 2.-Arc and spark spectra of lutecium $(Z=71)$

$c=$ Complex, hyperfine structure $(h f s)$.

$d=$ Double, may be $h f s$.

$e=$ Enhanced at electrode.

$h=$ Hazy.

$H=$ Very hazy. $l=$ Shaded or displaced to $\mid \mathrm{I}=$ Spectrum of singly ionized longer waves.

$B=B$ and head.

$p=$ Part of band structure.

Ins.

$\mathrm{II}=$ Spectrum of doubly ionized $\mathrm{LuO}=$ Molecular spectrum .

\begin{tabular}{|c|c|c|c|c|c|c|c|c|c|c|c|}
\hline \multirow{2}{*}{$\lambda_{\text {air }} A$} & \multicolumn{2}{|c|}{$\begin{array}{l}\text { Intensity and } \\
\text { character }\end{array}$} & \multirow{2}{*}{$\begin{array}{l}\text { Spec- } \\
\text { trum }\end{array}$} & \multirow{2}{*}{$\lambda_{\mathrm{air}} \mathrm{A}$} & \multicolumn{2}{|c|}{$\begin{array}{l}\text { Intensity and } \\
\text { character }\end{array}$} & \multirow{2}{*}{$\begin{array}{l}\text { Spec- } \\
\text { trum }\end{array}$} & \multirow{2}{*}{$\lambda_{\mathrm{Bir}} \mathrm{A}$} & \multicolumn{2}{|c|}{$\begin{array}{l}\text { Intensity and } \\
\text { character }\end{array}$} & \multirow{2}{*}{$\begin{array}{l}\text { Spec- } \\
\text { trum }\end{array}$} \\
\hline & Arc & Spark & & & Arc & Spark & & & Arc & Spark & \\
\hline $\begin{array}{l}2065.42 \\
2070.66 \\
2071.95 \\
2085.69 \\
2086.06\end{array}$ & & $\begin{array}{r}30 h \\
3 h \\
2 \mathrm{Si} \\
5 \\
2\end{array}$ & $\begin{array}{l}\text { II } \\
\text { II } \\
\text { II } \\
\text { II } \\
\text { II }\end{array}$ & $\begin{array}{l}2179.92 \\
2180.79 \\
2182.96 \\
2183.94 \\
2184.80\end{array}$ & & $\begin{array}{l}1 \\
5 \\
1 \\
1 \\
6\end{array}$ & $\begin{array}{l}\text { II } \\
\text { II } \\
\text { II } \\
\text { II } \\
\text { II }\end{array}$ & $\begin{array}{l}2306.38 \\
2307.54 \\
2310.66 \\
2313.03 \\
2313.98\end{array}$ & & $\begin{array}{r}1 \\
1 \\
2 h \\
5 \\
15 h l\end{array}$ & $\begin{array}{l}\text { II } \\
\text { II } \\
\text { II } \\
\text { II } \\
\text { II }\end{array}$ \\
\hline $\begin{array}{l}2086.45 \\
2087.50 \\
2092.14 \\
2098.94 \\
2099.54\end{array}$ & & $\begin{array}{r}4 \\
5 \\
6 \\
1 \\
10 h\end{array}$ & $\begin{array}{l}\text { II } \\
\text { II } \\
\text { II } \\
\text { II } \\
\text { II }\end{array}$ & $\begin{array}{l}2185.42 \\
2190.14 \\
2190.77 \\
2191.37 \\
2194.80\end{array}$ & 6 & $\begin{array}{r}2 \\
6 \\
4 \\
60 \\
7\end{array}$ & $\begin{array}{l}\text { II } \\
\text { II } \\
\text { II } \\
\text { II } \\
\text { II }\end{array}$ & $\begin{array}{l}2322.61 \\
2333.04 \\
2333.41 \\
2334.38 \\
2337.05\end{array}$ & & $\begin{array}{r}1 \\
1 \\
1 \\
2 \mathrm{Yb} ? \\
3\end{array}$ & $\begin{array}{l}\text { II } \\
\text { II } \\
\text { II } \\
\text { II } \\
\text { II }\end{array}$ \\
\hline $\begin{array}{l}2100.40 \\
2101.32 \\
2104.40 \\
2107.84 \\
2109.14\end{array}$ & & $\begin{array}{r}2 \\
1 \\
40 \\
4 \\
5\end{array}$ & $\begin{array}{l}\text { II } \\
\text { II } \\
\text { II } \\
\text { II } \\
\text { II }\end{array}$ & $\begin{array}{l}2195.54 \\
2196.54 \\
2196.98 \\
2197.90 \\
2200.31\end{array}$ & $\begin{array}{c}30 \\
-1 . \\
\end{array}$ & $\begin{array}{r}100 \\
2 \\
4 \\
2 \\
6\end{array}$ & $\begin{array}{l}\text { II } \\
\text { II } \\
\text { II } \\
\text { II } \\
\text { II }\end{array}$ & $\begin{array}{l}2338.43 \\
2341.19 \\
2343.07 \\
2345.50 \\
2348.60\end{array}$ & 2 & $\begin{array}{r}1 \\
3 \\
15 \\
1 \\
2\end{array}$ & $\begin{array}{l}\text { II } \\
\text { II } \\
\text { II } \\
\text { II } \\
\text { II }\end{array}$ \\
\hline $\begin{array}{l}2110.22 \\
2110.37 \\
2117.14 \\
2117.82 \\
2122.76\end{array}$ & & $\begin{array}{r}7 \\
4 \\
4 \\
2 \mathrm{Yb} ? \\
6 h\end{array}$ & $\begin{array}{l}\text { II } \\
\text { II } \\
\text { II } \\
\text { II } \\
\text { II }\end{array}$ & $\begin{array}{l}2204.60 \\
2206.82 \\
2207.64 \\
2216.70 \\
2217.32\end{array}$ & & $\begin{array}{c}2 h \\
5 h \\
2 h \\
1 \\
3 h\end{array}$ & $\begin{array}{l}\text { II } \\
\text { II } \\
\text { II } \\
\text { II } \\
\text { II }\end{array}$ & $\begin{array}{l}2349.14 \\
2350.43 \\
2350.73 \\
2351.88 \\
2352.76\end{array}$ & & $\begin{array}{r}2 h \\
3 \\
1 \\
2 h \\
1\end{array}$ & $\begin{array}{l}\text { II } \\
\text { II } \\
\text { II } \\
\text { II } \\
\text { II }\end{array}$ \\
\hline $\begin{array}{l}2124.13 \\
2124.64 \\
2127.43 \\
2128.39 \\
2131.66\end{array}$ & & $\begin{array}{r}3 \mathrm{Yb} ? \\
2 \\
4 \\
5 \\
6\end{array}$ & $\begin{array}{l}\text { II } \\
\text { II } \\
\text { II } \\
\text { II } \\
\text { II }\end{array}$ & $\begin{array}{l}2220.96 \\
2221.74 \\
2224.02 \\
2236.17 \\
2239.39\end{array}$ & - & $\begin{array}{r}1 \\
2 \\
6 h \\
150 h \\
1\end{array}$ & $\begin{array}{l}\text { II } \\
\text { II } \\
\text { II } \\
\text { III } \\
\text { II }\end{array}$ & $\begin{array}{l}2357.28 \\
2359.38 \\
2363.18 \\
2367.77 \\
2368.22\end{array}$ & & $\begin{array}{l}4 \\
2 \\
1 \\
1 \\
2\end{array}$ & $\begin{array}{l}\text { II } \\
\text { II } \\
\text { II } \\
\text { II } \\
\text { II }\end{array}$ \\
\hline $\begin{array}{l}2132.31 \\
2135.18 \\
2137.72 \\
2139.19 \\
2139.76\end{array}$ & & $\begin{array}{r}5 \\
5 \\
8 \mathrm{Yb} ? \\
6 \\
1\end{array}$ & $\begin{array}{l}\text { II } \\
\text { II } \\
\text { II } \\
\text { II } \\
\text { II }\end{array}$ & $\begin{array}{l}2249.53 \\
2260.40 \\
2260.79 \\
2263.98 \\
2264.63\end{array}$ & & $\begin{array}{r}2 h \\
4 \\
2 \\
3 \\
2\end{array}$ & $\begin{array}{l}\text { II } \\
\text { II } \\
\text { II } \\
\text { II } \\
\text { II }\end{array}$ & $\begin{array}{l}2369.08 \\
2380.57 \\
2381.69 \\
2385.20 \\
2387.45\end{array}$ & & $\begin{array}{r}5 \\
2 \\
30 h \\
1 \\
1\end{array}$ & $\begin{array}{l}\text { II } \\
\text { II } \\
\text { II, III? } \\
\text { II } \\
\text { II }\end{array}$ \\
\hline $\begin{array}{l}2140.53 \\
2143.84 \\
2146.96 \\
2147.59 \\
2150.51\end{array}$ & & $\begin{array}{r}2 h \\
3 \\
2 h \\
2 h \\
2\end{array}$ & $\begin{array}{l}\text { II } \\
\text { II } \\
\text { II } \\
\text { II } \\
\text { II }\end{array}$ & $\begin{array}{l}2269.65 \\
2269.94 \\
2270.61 \\
2270.95 \\
2271.97\end{array}$ & \begin{tabular}{l}
-1 \\
$-\cdots-1$ \\
\hdashline$-\cdots$
\end{tabular} & $\begin{array}{r}1 \\
1 \\
4 h \\
1 \\
1 \\
1\end{array}$ & $\begin{array}{l}\text { II } \\
\text { II } \\
\text { II } \\
\text { II } \\
\text { II }\end{array}$ & $\begin{array}{l}2388.69 \\
2390.23 \\
2392.19 \\
2394.11 \\
2394.77\end{array}$ & 30 & $\begin{array}{r}2 \\
2 \\
100 \\
1 \\
1\end{array}$ & $\begin{array}{l}\text { II } \\
\text { II } \\
\text { II } \\
\text { II } \\
\text { II }\end{array}$ \\
\hline $\begin{array}{l}2151.68 \\
2154.36 \\
2155.92 \\
2156.73 \\
2158.04\end{array}$ & & $\begin{array}{l}1 \\
7 \\
3 \\
4 \\
2\end{array}$ & $\begin{array}{l}\text { II } \\
\text { II } \\
\text { II } \\
\text { II } \\
\text { II }\end{array}$ & $\begin{array}{l}2276.94 \\
2283.99 \\
2291.74 \\
2293.34 \\
2293.78\end{array}$ & \begin{tabular}{|c|}
8 \\
-1 \\
-1 \\
-2 \\
\end{tabular} & $\begin{array}{r}20 \\
3 \mathrm{Yb} ? \\
1 \\
1 \\
2\end{array}$ & $\begin{array}{l}\text { II } \\
\text { II } \\
\text { II } \\
\text { II } \\
\text { II }\end{array}$ & $\begin{array}{l}2397.62 \\
2398.28 \\
2399.14 \\
2401.58 \\
2403.69\end{array}$ & $\begin{array}{c}10 \\
10 \\
-10\end{array}$ & $\begin{array}{r}1 \\
1 \\
50 \\
2 \\
4 h\end{array}$ & $\begin{array}{l}\text { II } \\
\text { II } \\
\text { II } \\
\text { II } \\
\text { II }\end{array}$ \\
\hline $\begin{array}{l}2159.91 \\
2164.33 \\
2173.30 \\
2176.93 \\
2178.02\end{array}$ & -1 & $\begin{array}{r}2 \\
15 \\
5 \mathrm{Yb} ? \\
4 \\
8\end{array}$ & $\begin{array}{l}\text { II } \\
\text { II } \\
\text { II } \\
\text { II } \\
\text { II }\end{array}$ & $\begin{array}{l}2297.41 \\
2298.99 \\
2301.71 \\
2302.84 \\
2303.30\end{array}$ & 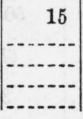 & $\begin{array}{r}100 \\
1 \\
1 \\
1 \\
2 \mathrm{Yb} ?\end{array}$ & $\begin{array}{l}\text { II } \\
\text { II } \\
\text { II } \\
\text { II } \\
\text { II }\end{array}$ & $\begin{array}{l}2405.76 \\
2406.21 \\
2406.82 \\
2408.13 \\
2415.92\end{array}$ & 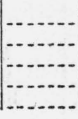 & $\begin{array}{l}1 \\
2 \\
2 \\
1 \\
5\end{array}$ & $\begin{array}{l}\text { II } \\
\text { II } \\
\text { II } \\
\text { II } \\
\text { II }\end{array}$ \\
\hline
\end{tabular}

See footnotes at end of table. 
TABLE 2.-Arc and spark spectra of lutecium $(Z=\gamma 1)$-Continued

\begin{tabular}{|c|c|c|c|c|c|c|c|c|c|c|c|}
\hline \multirow{2}{*}{$\lambda_{\text {air }} \mathbf{A}$} & \multicolumn{2}{|c|}{$\begin{array}{c}\text { Intensity and } \\
\text { character }\end{array}$} & \multirow{2}{*}{$\begin{array}{l}\text { Spec- }- \\
\text { trum }\end{array}$} & \multirow{2}{*}{$\lambda_{\text {air }} \mathbf{A}$} & \multicolumn{2}{|c|}{$\begin{array}{l}\text { Intensity and } \\
\text { character }\end{array}$} & \multirow{2}{*}{$\begin{array}{l}\text { Spec- } \\
\text { trum }\end{array}$} & \multirow{2}{*}{$\lambda_{\text {gir }} \mathbf{A}$} & \multicolumn{2}{|c|}{$\begin{array}{l}\text { Intensity and } \\
\text { character }\end{array}$} & \multirow{2}{*}{$\begin{array}{l}\text { Spec- } \\
\text { trum }\end{array}$} \\
\hline & Arc & Spark & & & Arc & Spark & & & Are & Spark & \\
\hline $\begin{array}{l}2419.21 \\
2421.42 \\
2421.97 \\
2424.90 \\
2427.21\end{array}$ & \begin{tabular}{l}
8 \\
$\cdots-$. \\
\hdashline.- \\
\hdashline-
\end{tabular} & $\begin{array}{r}40 \\
4 \mathrm{Yb} ? \\
1 \\
1 \\
3\end{array}$ & $\begin{array}{l}\text { II } \\
\text { II } \\
\text { II } \\
\text { II } \\
\text { II }\end{array}$ & $\begin{array}{l}2555.82 \\
2556.91 \\
2558.42 \\
2561.80 \\
2563.52\end{array}$ & $1 e$ & $\begin{array}{r}2 \\
2 \\
6 h \\
6 h \\
80 h\end{array}$ & $\begin{array}{r}\text { II } \\
\text { II } \\
\text { II } \\
\text { II } \\
\text { III }\end{array}$ & $\begin{array}{l}2835.25 \\
2845.13 \\
2845.87 \\
2847.51 \\
2855.36\end{array}$ & 40 & $\begin{array}{r}10 h d ? \\
2 \\
1 h \\
120 \\
2\end{array}$ & $\begin{array}{l}\text { II } \\
\text { I } \\
\text { II } \\
\text { II } \\
\text { II }\end{array}$ \\
\hline $\begin{array}{l}2430.26 \\
2433.02 \\
2434.25 \\
2435.12 \\
2445.83\end{array}$ & $\begin{array}{l}-- \\
-- \\
\end{array}$ & $\begin{array}{r}15 \\
1 \\
2 \\
1 \mathrm{Yb} ? \\
1\end{array}$ & $\begin{array}{l}\text { II } \\
\text { II } \\
\text { II } \\
\text { II } \\
\text { II }\end{array}$ & $\begin{array}{l}2571.23 \\
2573.55 \\
2578.79 \\
2582.13 \\
2590.03\end{array}$ & \begin{tabular}{r}
30 \\
\hdashline 40 \\
3
\end{tabular} & $\begin{array}{r}100 \\
2 \\
120 \\
20 \\
3\end{array}$ & $\begin{array}{l}\text { II } \\
\text { II } \\
\text { II } \\
\text { II } \\
\text { II }\end{array}$ & $\begin{array}{l}2860.31 \\
2860.94 \\
2862.74 \\
2863.61 \\
2876.54\end{array}$ & 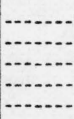 & $\begin{array}{r}3 \\
1 \\
1 \\
1 h \\
2 h\end{array}$ & $\begin{array}{l}\text { II } \\
\text { II } \\
\text { II } \\
\text { II } \\
\text { II }\end{array}$ \\
\hline $\begin{array}{l}2449.75 \\
2449.96 \\
2451.60 \\
2454.87 \\
2455.60\end{array}$ & & $\begin{array}{l}2 \\
2 \\
1 \\
2 \\
4\end{array}$ & $\begin{array}{l}\text { II } \\
\text { II } \\
\text { II } \\
\text { II } \\
\text { II }\end{array}$ & $\begin{array}{l}2590.58 \\
2592.27 \\
2592.34 \\
2603.33 \text {. } \\
2607.32\end{array}$ & \begin{tabular}{c}
$2 h$ \\
\hdashline $10 d, e$ \\
\hdashline
\end{tabular} & $\begin{array}{r}2 \\
? \\
5 h \\
300 h \\
4 h\end{array}$ & $\begin{array}{l}\text { II } \\
\text { II } \\
\text { II } \\
\text { III } \\
\text { II }\end{array}$ & $\begin{array}{l}2879.02 \\
2885.14 \\
2886.04 \\
2894.84 \\
2900.30\end{array}$ & $\begin{array}{r}40 h \\
3 \\
60 \\
50\end{array}$ & \begin{tabular}{r}
$2 h$ \\
3 \\
\hdashline 200 \\
150
\end{tabular} & $\begin{array}{r}\text { II } \\
\mathbf{I} \\
\mathbf{I} \\
\text { II } \\
\text { II }\end{array}$ \\
\hline $\begin{array}{l}2456.50 \\
2457.56 \\
2459.64 \\
2463.95 \\
2464.82\end{array}$ & - & $\begin{array}{c}2 \\
5 h \\
8 \\
3 \\
?\end{array}$ & $\begin{array}{l}\text { II } \\
\text { II } \\
\text { II } \\
\text { II } \\
\text { I? }\end{array}$ & $\begin{array}{l}2612.86 \\
2613.40 \\
2615.42 \\
2619.26 \\
2621.88\end{array}$ & $\begin{array}{r}3 h \\
30 \\
100 \\
30 \\
1\end{array}$ & $\begin{array}{r}? \\
100 \\
250 \\
100 \\
?\end{array}$ & $\begin{array}{l}\text { I? } \\
\text { II } \\
\text { II } \\
\text { II } \\
\text { I? }\end{array}$ & $\begin{array}{l}2903.05 \\
2905.92 \\
2911.39 \\
2912.70 \\
2920.69\end{array}$ & $\begin{array}{r}20 \\
100 \\
15 h\end{array}$ & $\begin{array}{r}1 \\
1 \\
300 \\
1\end{array}$ & $\begin{array}{l}\text { I } \\
\text { II } \\
\text { II } \\
\text { I } \\
\text { II }\end{array}$ \\
\hline $\begin{array}{l}2467.68 \\
2469.27 \\
2470.27 \\
2470.81 \\
2472.50\end{array}$ & & $\begin{array}{r}2 \\
40 \\
1 \\
1 \\
2 h\end{array}$ & $\begin{array}{l}\text { II } \\
\text { II } \\
\text { II } \\
\text { II } \\
\text { II }\end{array}$ & $\begin{array}{l}2624.58 \\
2633.72 \\
2634.75 \\
2644.56 \\
2652.48\end{array}$ & $\cdots$ & $\begin{array}{r}1 \\
2 \\
1 \\
2 h \\
1\end{array}$ & $\begin{array}{l}\text { II } \\
\text { II } \\
\text { II } \\
\text { II } \\
\text { II }\end{array}$ & $\begin{array}{l}2923.37 \\
2931.53 \\
2939.08 \\
2946.39 \\
2949.73\end{array}$ & $\begin{array}{c}7 h \\
20 h\end{array}$ & \begin{tabular}{r}
1 \\
\hdashline $2 h$ \\
$2 h$ \\
1
\end{tabular} & $\begin{array}{l}\text { II } \\
\text { I } \\
\text { II } \\
\text { II } \\
\text { I }\end{array}$ \\
\hline $\begin{array}{l}2475.34 \\
2481.72 \\
2487.96 \\
2488.28 \\
2489.24\end{array}$ & $\begin{array}{l}20 \\
-. . \\
-. .\end{array}$ & $\begin{array}{r}3 \\
100 \\
4 \\
1 \\
2\end{array}$ & $\begin{array}{l}\text { II } \\
\text { II } \\
\text { II } \\
\text { II } \\
\text { II }\end{array}$ & $\begin{array}{l}2653.34 \\
2657.80 \\
2660.46 \\
2670.78 \\
2677.25\end{array}$ & $\begin{array}{r}50 \\
5 h \\
10 h\end{array}$ & $\begin{array}{r}2 h \\
150 \\
2 \\
--\end{array}$ & $\begin{array}{c}\text { II } \\
\text { II } \\
\text { II } \\
\text { I } \\
\text { I }\end{array}$ & $\begin{array}{l}2951.69 \\
2955.78 \\
2960.10 \\
2963.32 \\
2969.82\end{array}$ & $\begin{array}{r}20 \\
2 \\
-50 \\
30\end{array}$ & $\begin{array}{r}80 \\
60 h l \\
2 h \\
150 \\
100\end{array}$ & $\begin{array}{l}\text { II } \\
\text { II } \\
\text { II } \\
\text { II } \\
\text { II }\end{array}$ \\
\hline $\begin{array}{l}2489.83 \\
2493.84 \\
2494.64 \\
2495.08 \\
2495.52\end{array}$ & & $\begin{array}{l}2 \\
2 \\
6 \\
1 \\
2\end{array}$ & $\begin{array}{l}\text { II } \\
\text { II } \\
\text { II } \\
\text { II } \\
\text { II }\end{array}$ & $\begin{array}{l}2677.77 \\
2685.08 \\
2685.54 \\
2688.09 \\
2692.34\end{array}$ & $\begin{array}{c}1 \\
50 h \\
10 h \\
5 h\end{array}$ & $\begin{array}{r}10 h \\
3 \\
1 \\
2 \\
-.\end{array}$ & $\begin{array}{r}\text { II } \\
\text { I } \\
\text { I } \\
\text { II } \\
\text { I }\end{array}$ & $\begin{array}{l}2975.67 \\
2978.50 \\
2985.85 \\
2989.27 \\
2992.48\end{array}$ & 50 & $\begin{array}{r}1 \\
1 \\
3 h \mathrm{Yb}^{2} \\
4 \\
1\end{array}$ & $\begin{array}{l}\text { II } \\
\text { II } \\
\text { II } \\
\text { I } \\
\text { II }\end{array}$ \\
\hline $\begin{array}{l}2495.86 \\
2496.83 \\
2497.83 \\
2498.99 \\
2500.23\end{array}$ & & $\begin{array}{r}1 \\
2 \\
2 \\
3 \\
1 h \\
1 h\end{array}$ & $\begin{array}{l}\text { II } \\
\text { II } \\
\text { II } \\
\text { II } \\
\text { II }\end{array}$ & $\begin{array}{l}2696.95 \\
2697.45 \\
2699.74 \\
2701.71 \\
2703.13\end{array}$ & $\begin{array}{l}40 \\
7 h\end{array}$ & $\begin{array}{r}2 \\
2 \\
3 \\
150 \\
-. .\end{array}$ & $\begin{array}{l}\text { II } \\
\text { II } \\
\text { II } \\
\text { II } \\
\text { I }\end{array}$ & $\begin{array}{l}2995.84 \\
3003.65 \\
3016.37 \\
3020.54 \\
3027.29\end{array}$ & $\begin{array}{r}40 \\
4\end{array}$ & $\begin{array}{r}15 h l \\
3 h \\
15 h l \\
100 \\
8\end{array}$ & $\begin{array}{l}\text { II } \\
\text { II } \\
\text { II } \\
\text { II } \\
\text { II }\end{array}$ \\
\hline $\begin{array}{l}2500.86 \\
2509.04 \\
2511.37 \\
2512.87 \\
2513.99\end{array}$ & & $\begin{array}{r}1 \\
3 \mathrm{Tm} ? \\
1 \\
2 \\
1\end{array}$ & $\begin{array}{l}\text { I } \\
\text { II } \\
\text { II } \\
\text { II } \\
\text { II }\end{array}$ & $\begin{array}{l}2707.66 \\
2715.38 \\
2715.91 \\
2719.09 \\
2724.81\end{array}$ & $\begin{array}{r}4 h \\
10 h\end{array}$ & \begin{tabular}{c}
1 \\
1 \\
\hdashline$?$ \\
4
\end{tabular} & $\begin{array}{r}\text { II } \\
\text { II } \\
\text { I } \\
\text { I } \\
\text { II }\end{array}$ & $\begin{array}{l}3040.04 \\
3044.18 \\
3047.36 \\
3055.70 \\
3056.72\end{array}$ & $\begin{array}{r}3 h \\
1 \\
50\end{array}$ & $\begin{array}{r}20 h l \\
1 \\
2 \\
100\end{array}$ & $\begin{array}{l}\text { II } \\
\text { II } \\
\text { I } \\
\text { II } \\
\text { II }\end{array}$ \\
\hline $\begin{array}{l}2514.41 \\
2518.04 \\
2518.77 \\
2519.05 \\
2520.53\end{array}$ & & $\begin{array}{l}1 \\
4 \\
1 \\
1 \\
2\end{array}$ & $\begin{array}{l}\text { II } \\
\text { II } \\
\text { II } \\
\text { II } \\
\text { II }\end{array}$ & $\begin{array}{l}2728.95 \\
2738.17 \\
2749.70 \\
2754.17 \\
2765.74\end{array}$ & $\begin{array}{r}40 \\
2 \\
-40 \\
20\end{array}$ & $\begin{array}{r}2 \\
25 h l \\
2 h d ? \\
120 \\
3\end{array}$ & $\begin{array}{l}\text { I } \\
\text { II } \\
\text { II } \\
\text { II } \\
\text { I }\end{array}$ & $\begin{array}{l}3057.90 \\
3062.65 \\
3063.51 \\
3067.83 \\
3077.60\end{array}$ & $\begin{array}{c}3 e \\
-20 h \\
100\end{array}$ & $\begin{array}{r}150 h \\
2 h \\
3 h \\
200\end{array}$ & $\begin{array}{r}\text { III } \\
\text { II } \\
\text { I } \\
\text { II } \\
\text { II }\end{array}$ \\
\hline $\begin{array}{l}2523.27 \\
2526.83 \\
2528.59 \\
2529.84 \\
2530.55\end{array}$ & & $\begin{array}{r}1 \\
10 h \\
5 h \\
1 \\
8 h\end{array}$ & $\begin{array}{l}\text { II } \\
\text { II } \\
\text { II } \\
\text { II } \\
\text { II }\end{array}$ & $\begin{array}{l}2772.58 \\
2778.52 \\
2781.47 \\
2784.80 \\
2785.70\end{array}$ & $\begin{array}{c}5 e \\
4 h \bar{h}\end{array}$ & $\begin{array}{r}150 h \\
1 \\
8 h \\
1\end{array}$ & $\begin{array}{r}\text { III } \\
\text { II } \\
\text { I } \\
\text { II } \\
\text { II }\end{array}$ & $\begin{array}{l}3080.11 \\
3081.47 \\
3089.02 \\
3101.99 \\
3104.98\end{array}$ & $\begin{array}{r}15 \\
80 \\
-0.9 \\
\end{array}$ & $\begin{array}{r}2 \\
8 \\
8 h \\
4 h l \\
25 h l\end{array}$ & $\begin{array}{l}\text { I } \\
\text { I } \\
\text { II } \\
\text { II } \\
\text { II }\end{array}$ \\
\hline $\begin{array}{l}2534.74 \\
2536.95 \\
2538.12 \\
2539.41 \\
2542.35\end{array}$ & 2 & $\begin{array}{r}2 \\
20 \\
3 \mathrm{Yb} ? \\
1 \\
?\end{array}$ & $\begin{array}{l}\text { II } \\
\text { II } \\
\text { II } \\
\text { II } \\
\text { I? }\end{array}$ & $\begin{array}{l}2787.44 \\
2796.63 \\
2817.95 \\
2819.50 \\
2821.23\end{array}$ & \begin{tabular}{r}
25 \\
\hdashline $2 e$ \\
-2
\end{tabular} & $\begin{array}{r}1 \\
100 \\
1 \\
6 h l \\
50 h l\end{array}$ & $\begin{array}{c}\text { II } \\
\text { II } \\
\text { II } \\
\text { II } \\
\text { III? }\end{array}$ & $\begin{array}{l}3115.32 \\
3118.43 \\
3161.62 \\
3167.33 \\
3171.36\end{array}$ & $\begin{array}{r}2 \\
40 \\
11 \\
40\end{array}$ & $\begin{array}{r}1 \\
5 \\
10 h l \\
15 h l \\
5\end{array}$ & $\begin{array}{l}\text { I? } \\
\text { I } \\
\text { II } \\
\text { II } \\
\text { I }\end{array}$ \\
\hline $\begin{array}{l}2546.32 \\
2546.87 \\
2548.63 \\
2549.52 \\
2550.67\end{array}$ & $20 \bar{h}$ & $\begin{array}{r}5 h \\
9 h \\
10 h \\
3\end{array}$ & $\begin{array}{r}\text { II } \\
\text { II } \\
\text { II } \\
\text { I } \\
\text { II }\end{array}$ & $\begin{array}{l}2826.82 \\
2828.95 \\
2829.42 \\
2830.79 \\
2834.35\end{array}$ & \begin{tabular}{|c|c|}
$\ldots$ \\
-1
\end{tabular} & $\begin{array}{r}6 h l \\
1 \\
3 h \\
1 \\
40 h\end{array}$ & $\begin{array}{l}\text { II } \\
\text { II } \\
\text { II } \\
\text { II } \\
\text { II }\end{array}$ & $\begin{array}{l}3180.27 \\
3183.73 \\
3191.80 \\
3198.12 \\
3214.31\end{array}$ & $\begin{array}{r}3 \\
3 \\
40\end{array}$ & $\begin{array}{r}1 h \\
6 \\
60 h l \\
80 \\
1 \mathrm{Yb} ?\end{array}$ & $\begin{array}{l}\text { II } \\
\text { II } \\
\text { II } \\
\text { II } \\
\text { II }\end{array}$ \\
\hline
\end{tabular}

See footnotes at end of table. 
TABLE 2.-Arc and spark spectra of lutecium $(Z=71)$-Continued

\begin{tabular}{|c|c|c|c|c|c|c|c|c|c|c|c|}
\hline \multirow{2}{*}{$\lambda_{\text {sir }} \mathbf{A}$} & \multicolumn{2}{|c|}{$\begin{array}{c}\text { Intensity and } \\
\text { character }\end{array}$} & \multirow{2}{*}{$\begin{array}{l}\text { Spec- } \\
\text { trum }\end{array}$} & \multirow{2}{*}{$\lambda_{\mathrm{sir}} \mathrm{A}$} & \multicolumn{2}{|c|}{$\begin{array}{l}\text { Intensity and } \\
\text { character }\end{array}$} & \multirow{2}{*}{$\begin{array}{l}\text { Spec- } \\
\text { trum }\end{array}$} & \multirow{2}{*}{$\lambda_{\mathrm{Bir}} \mathrm{A}$} & \multicolumn{2}{|c|}{$\begin{array}{l}\text { Intensity and } \\
\text { character }\end{array}$} & \multirow{2}{*}{$\begin{array}{l}\text { Spec- } \\
\text { trum }\end{array}$} \\
\hline & Arc & Spark & & & Arc & Spark & & & Arc & Spark & \\
\hline $\begin{array}{l}19.09 \\
22.57 \\
42.93 \\
45.93 \\
46.71\end{array}$ & $\cdots$ & $\begin{array}{l}3 h \\
8 h \\
4 h \\
2 h \\
2 h\end{array}$ & $\begin{array}{l}\text { II } \\
\text { II } \\
\text { II } \\
\text { II } \\
\text { II }\end{array}$ & $\begin{array}{l}3829.07 \\
3841.18 \\
3843.61 \\
3853.29 \\
3870.88\end{array}$ & $\begin{array}{r}10 h \\
100 \\
15 h \\
10 \\
5\end{array}$ & 8 & $\begin{array}{l}\text { I } \\
\text { I } \\
\text { I } \\
\text { I } \\
\text { I }\end{array}$ & $\begin{array}{l}4521.08 \\
4525.48 \\
4533.39 \\
4553.47 \\
4560.95\end{array}$ & $\begin{array}{r}2 h \\
2 h \\
5 B ? \\
2 p ? \\
8 B l\end{array}$ & $\because$ & $\begin{array}{l}\text { I } \\
\text { I } \\
\text { LuO? } \\
\text { I } \\
\text { LuO }\end{array}$ \\
\hline $\begin{array}{l}3249.47 \\
3251.95 \\
3254.31 \\
3265.00 \\
3265.62\end{array}$ & $\begin{array}{r}50 \\
2\end{array}$ & $\begin{array}{r}4 \\
1 h \\
150 \\
10 h l\end{array}$ & $\begin{array}{r}\text { II } \\
\text { II } \\
\text { II } \\
\text { II } \\
\text { I }\end{array}$ & $\begin{array}{l}3874.61 \\
3876.65 \\
3888.38 \\
3899.54 \\
3911.77\end{array}$ & $\begin{array}{r}4 \\
50 c \\
3 h\end{array}$ & $\begin{array}{r}100 c \\
1 h \\
3 h\end{array}$ & $\begin{array}{l}\text { I } \\
\text { II } \\
\text { II } \\
\text { II } \\
\text { I }\end{array}$ & $\begin{array}{l}4569.5 \\
4575.31 \\
4585.17 \\
4586.93 \\
4590.68\end{array}$ & $\begin{array}{r}5 B l \\
5 B l \\
1 p ? \\
2 \\
3 B ?\end{array}$ & 6 & $\begin{array}{l}\text { LuO } \\
\text { LuO } \\
\text { I } \\
\text { II } \\
\text { LuO? }\end{array}$ \\
\hline $\begin{array}{l}3278.97 \\
3280.50 \\
3281.74 \\
3303.75 \\
3305.68\end{array}$ & $\begin{array}{r}50 \\
10 \\
60 \\
-2\end{array}$ & $\begin{array}{r}5 \\
1 h \\
1 h \\
? \mathrm{Yb} ?\end{array}$ & $\begin{array}{c}\text { I } \\
\text { I } \\
\text { I } \\
\text { II } \\
\text { I? }\end{array}$ & $\begin{array}{l}3918.86 \\
3925.30 \\
3926.62 \\
3937.61 \\
3968.46\end{array}$ & $\begin{array}{r}4 \\
20 \\
2 \\
-50 ?\end{array}$ & $\begin{array}{r}1 \\
5 h l \\
\mathrm{Ca}^{+}\end{array}$ & $\begin{array}{r}\text { I } \\
\text { I } \\
\text { I } \\
\text { II } \\
\text { I }\end{array}$ & $\begin{array}{l}4602.04 \\
4602.60 \\
4605.39 \\
4643.29 \\
4645.47\end{array}$ & $\begin{array}{r}1 p ? \\
3 h \\
10 h \\
8 h \\
25 h\end{array}$ & 2 & $\begin{array}{l}\mathbf{I} \\
\mathbf{I} \\
\mathbf{I} \\
\mathbf{I} \\
\mathbf{I}\end{array}$ \\
\hline $\begin{array}{l}3312.11 \\
3319.63 \\
3332.61 \\
3338.62 \\
3359.56\end{array}$ & $\begin{array}{r}100 \\
150\end{array}$ & $\begin{array}{r}10 \\
3 h \\
8 h \\
1 \\
15\end{array}$ & $\begin{array}{r}\text { I } \\
\text { II } \\
\text { II } \\
\text { II } \\
\text { I }\end{array}$ & $\begin{array}{l}3981.01 \\
3991.38 \\
4030.86 \\
4033.02 \\
4064.45\end{array}$ & $\begin{array}{r}3 \\
4 \text { Ga? } \\
25\end{array}$ & $\frac{2 h}{5 h l}$ & $\begin{array}{r}\text { II } \\
\text { I } \\
\text { II } \\
\text { I } \\
\text { I }\end{array}$ & $\begin{array}{l}4648.21 \\
4648.85 \\
4651.94 \\
4654.03 \\
4656.49\end{array}$ & $\begin{array}{r}25 h \\
25 h \\
2 p ? \\
15 B l \\
2 p ?\end{array}$ & $\begin{array}{c}2 \\
? \\
- \\
-\end{array}$ & $\begin{array}{l}\text { I } \\
\text { I } \\
\text { L LuO }\end{array}$ \\
\hline $\begin{array}{l}3364.26 \\
3376.50 \\
3385.50 \\
3391.55 \\
3396.82\end{array}$ & $\begin{array}{r}100 \\
30 \\
10 \\
30\end{array}$ & $\begin{array}{r}1 \\
10 \\
4 \\
2 \\
1\end{array}$ & $\begin{array}{r}\text { II } \\
\text { I } \\
\text { I } \\
\text { I } \\
\text { I }\end{array}$ & $\begin{array}{l}4079.87 \\
4094.04 \\
4096.13 \\
4107.44 \\
4112.67\end{array}$ & $\begin{array}{r}1 h \\
10 \mathrm{~B} l \\
20 \mathrm{~B} l \\
3 p ? \\
5\end{array}$ & & $\begin{array}{c}\text { I } \\
\text { LuO } \\
\text { LuO } \\
\text { I } \\
\text { I }\end{array}$ & $\begin{array}{l}4658.02 \\
4659.03 \\
4661.75 \\
4672.31 \\
4675.29\end{array}$ & $\begin{array}{r}100 \\
10 \\
150 B l \\
120 B l \\
4 p ?\end{array}$ & $\begin{array}{r}15 \\
1 \\
15 B l \\
12 B l\end{array}$ & $\begin{array}{l}\text { I } \\
\text { I } \\
\text { LuO } \\
\text { I }\end{array}$ \\
\hline $\begin{array}{l}3397.07 \\
3418.42 \\
3423.30 \\
3454.77 \\
3472.48\end{array}$ & $\begin{array}{r}50 \\
3 \mathrm{Yb} ? \\
50\end{array}$ & $\begin{array}{r}200 \\
1 \bar{h} \\
3 h \\
150\end{array}$ & $\begin{array}{l}\text { II } \\
\text { I } \\
\text { II } \\
\text { II } \\
\text { II }\end{array}$ & $\begin{array}{l}4122.49 \\
4124.73 \\
4131.79 \\
4154.08 \\
4158.98\end{array}$ & $\begin{array}{r}15 \\
200 \\
10+p \\
40 \\
1\end{array}$ & $\begin{array}{r}2 \\
10 \\
\hdashline 3\end{array}$ & $\begin{array}{l}\text { I } \\
\text { I } \\
\text { I } \\
\text { I } \\
\text { I }\end{array}$ & $\begin{array}{l}4684.16 \\
4689.77 \\
4695.46 \\
4708.00 \\
4716.70\end{array}$ & $\begin{array}{r}100 B l \\
4+p \\
80 B l \\
60 B l \\
5\end{array}$ & $\begin{array}{c}10 B l \\
8 B \bar{l} \\
6 B l\end{array}$ & $\begin{array}{l}\text { LuO } \\
\text { I } \\
\text { LuO } \\
\text { LuO } \\
\text { I }\end{array}$ \\
\hline $\begin{array}{l}3491.92 \\
3507.39 \\
3508.42 \\
3518.89 \\
3521.18\end{array}$ & $\begin{array}{r}100 c \\
30 \\
1 \\
3\end{array}$ & $\begin{array}{r}3 h \\
150 \\
3\end{array}$ & $\begin{array}{c}\text { II } \\
\text { II } \\
\text { I } \\
\text { I } \\
\text { I }\end{array}$ & $\begin{array}{l}4167.50 \\
4184.25 \\
4223.09 \\
4223.99 \\
4235.51\end{array}$ & $\begin{array}{r}2 \\
100 \\
1 \\
2 \\
1\end{array}$ & $\begin{array}{r}200 \\
4\end{array}$ & $\begin{array}{r}\text { I } \\
\text { II } \\
\text { II } \\
\text { I } \\
\text { I }\end{array}$ & $\begin{array}{l}4720.86 \\
4726.20 \\
4733.50 \\
4735.00 \\
4748.38\end{array}$ & $\begin{array}{r}5 B \\
4 p ? \\
\hdashline 25 B l\end{array}$ & $\begin{array}{r}3 \bar{h} \\
2 \\
10 h l\end{array}$ & $\begin{array}{l}\text { LuO } \\
\text { I } \\
\text { II } \\
\text { LuO } \\
\text { II }\end{array}$ \\
\hline $\begin{array}{l}3525.94 \\
3546.39 \\
3553.10 \\
3554.43 \\
3567.84\end{array}$ & $\begin{array}{r}2 \\
7 \\
2 \\
50 \\
100\end{array}$ & $\begin{array}{r}150 \\
7\end{array}$ & $\begin{array}{r}\text { I } \\
\text { I } \\
\text { I } \\
\text { II } \\
\text { I }\end{array}$ & $\begin{array}{l}4239.30 \\
4241.9 \\
4252.53 \\
4262.02 \\
4266.40\end{array}$ & $\begin{array}{c}5 \mathrm{~B} l ? \\
10 B l \\
5 B ?\end{array}$ & $\begin{array}{r}\mathrm{Yb}^{+} \\
8 \\
2 h\end{array}$ & $\begin{array}{l}\text { LuO } \\
\text { LuO } \\
\text { LuO } \\
\text { II } \\
\text { II }\end{array}$ & $\begin{array}{l}4749.11 \\
4757.26 \\
4764.22 \\
4774.05 \\
4779.00\end{array}$ & $\begin{array}{r}10 B l \\
1 p ? \\
7 B l \\
1 p ? \\
1 \mathrm{Y} \text { b? }\end{array}$ & $\begin{array}{c}1 \\
-1 \\
-\end{array}$ & $\begin{array}{l}\text { LuO } \\
\text { I } \\
\text { LuO } \\
\text { I } \\
\text { I }\end{array}$ \\
\hline $\begin{array}{l}3580.26 \\
3596.34 \\
3620.31 \\
3623.99 \\
3628.90\end{array}$ & $\begin{array}{r}3 \\
6 \\
4 \\
20 \\
2\end{array}$ & 40 & $\begin{array}{r}\text { I } \\
\text { I } \\
\text { I } \\
\text { II } \\
\text { I }\end{array}$ & $\begin{array}{l}4277.50 \\
4281.03 \\
4295.97 \\
4296.09 \\
4309.57\end{array}$ & $\begin{array}{l}30 \\
40 \\
30 \\
15 \\
25\end{array}$ & $\begin{array}{l}3 \\
4 \\
3 \\
2 \\
2\end{array}$ & $\begin{array}{l}\mathbf{I} \\
\mathbf{I} \\
\mathbf{I} \\
\mathbf{I} \\
\mathbf{I}\end{array}$ & $\begin{array}{l}4780.11 \\
4785.42 \\
4798.00 \\
4810.52 \\
4815.05\end{array}$ & $\begin{array}{r}5 B \\
100 \\
3 B \\
2 \mathrm{Zn} ? \\
20\end{array}$ & 200 & $\begin{array}{l}\text { LuO } \\
\text { II } \\
\text { LuO } \\
\text { I } \\
\text { I }\end{array}$ \\
\hline $\begin{array}{l}3636.25 \\
3642.35 \\
3647.77 \\
3678.04 \\
3682.40\end{array}$ & $\begin{array}{r}25 \\
2 ? \\
100 \\
\hdashline 1 ?\end{array}$ & $\begin{array}{c}5 \\
3 h\end{array}$ & $\begin{array}{r}\text { I } \\
\text { I } \\
\text { I } \\
\text { II } \\
\text { I }\end{array}$ & $\begin{array}{l}4332.72 \\
4341.98 \\
4355.05 \\
4374.90 \\
4396.16\end{array}$ & $\begin{array}{r}10 \\
3 \\
3 \\
5 \\
2\end{array}$ & $\begin{array}{r}1 \\
30 h l \\
?\end{array}$ & $\begin{array}{c}\text { I } \\
\text { II } \\
\text { I } \\
\text { I } \\
\text { I? }\end{array}$ & $\begin{array}{l}4839.62 \\
4843.03 \\
4844.44 \\
4858.75 \\
4860.15\end{array}$ & $\begin{array}{r}50 c \\
2 \\
3 \\
2 \\
1 h\end{array}$ & $\begin{array}{r}100 c \\
5 \\
8\end{array}$ & $\begin{array}{l}\text { II } \\
\text { II } \\
\text { I } \\
\text { II } \\
\text { I }\end{array}$ \\
\hline $\begin{array}{l}3684.32 \\
3704.79 \\
3706.16 \\
3710.95 \\
3722.58\end{array}$ & \begin{tabular}{r}
15 \\
\hdashline 2 \\
3 \\
$3 ?$
\end{tabular} & $\begin{array}{c}1 \\
3 h\end{array}$ & $\begin{array}{r}\text { I } \\
\text { II } \\
\text { I } \\
\text { I } \\
\text { I }\end{array}$ & $\begin{array}{l}4397.31 \\
4404.86 \\
4416.45 \\
4420.96 \\
4430.48\end{array}$ & $\begin{array}{r}7 \\
5 ? \\
6 \\
15 c \\
30 c\end{array}$ & 2 & $\begin{array}{l}\text { I } \\
\text { I } \\
\text { I } \\
\text { I } \\
\text { I }\end{array}$ & $\begin{array}{l}4864.52 \\
4865.36 \\
4870.78 \\
4882.40 \\
4888.14\end{array}$ & $\begin{array}{r}1 h \\
4 \\
1 h \\
3 h \\
2 h\end{array}$ & 20 & $\begin{array}{l}\text { I } \\
\text { II } \\
\text { I } \\
\text { I } \\
\text { I }\end{array}$ \\
\hline $\begin{array}{l}3724.32 \\
3742.08 \\
3756.70 \\
3756.79 \\
3777.15\end{array}$ & $\begin{array}{r}2 h ? \\
2 \\
8 \\
6 \\
--\end{array}$ & $2 h$ & $\begin{array}{r}\text { I } \\
\text { I } \\
\text { I } \\
\text { I } \\
\text { II }\end{array}$ & $\begin{array}{l}4438.79 \\
4450.81 \\
4471.55 \\
4480.15 \\
4490.53\end{array}$ & $\begin{array}{r}4 \\
40 \\
7 \\
3 \\
2\end{array}$ & 2 & $\begin{array}{l}\text { I } \\
\text { I } \\
\text { I } \\
\text { I } \\
\text { I }\end{array}$ & $\begin{array}{l}4895.00 \\
4904.88 \\
4907.16 \\
4911.41 \\
4921.70\end{array}$ & $\begin{array}{r}1 h \\
60 \\
1 h \\
1 \\
3\end{array}$ & \begin{tabular}{c}
$-\overline{5}$ \\
\hdashline 8
\end{tabular} & $\begin{array}{l}\text { I } \\
\text { I } \\
\text { I } \\
\text { I } \\
\text { II }\end{array}$ \\
\hline $\begin{array}{l}3786.18 \\
3793.24 \\
3800.67 \\
3802.62 \\
3814.02\end{array}$ & $\begin{array}{l}4 \\
4 \\
5 \\
3 \\
2\end{array}$ & ו & $\begin{array}{l}\text { I } \\
\text { I } \\
\text { I } \\
\text { I } \\
\text { I }\end{array}$ & $\begin{array}{l}4498.85 \\
4505.22 \\
4508.91 \\
4517.71 \\
4518.57\end{array}$ & $\begin{array}{r}10 \\
1 \\
5 B ? \\
1 h \\
300 \mathrm{c}\end{array}$ & $\begin{array}{l}1 \\
5\end{array}$ & $\begin{array}{l}\text { I } \\
\text { II } \\
\text { LuO } \\
\text { I } \\
\text { I }\end{array}$ & $\begin{array}{l}4942.34 \\
4976.92 \\
4994.13 \circ \\
5001.14 \\
5057.60\end{array}$ & $\begin{array}{r}40 \\
1 h \\
250 c \\
100 \\
15\end{array}$ & $\begin{array}{r}3 \\
400 c \\
? \\
1\end{array}$ & \begin{tabular}{|l|} 
I \\
I \\
II \\
I \\
I
\end{tabular} \\
\hline
\end{tabular}

See footnotes at end of table. 
TABLE 2.-Arc and spark spectra of lutecium $(Z=71)$-Continued

\begin{tabular}{|c|c|c|c|c|c|c|c|c|c|c|c|}
\hline \multirow{2}{*}{$\lambda_{\mathrm{air}} \mathrm{A}$} & \multicolumn{2}{|c|}{$\begin{array}{l}\text { Intensity and } \\
\text { character }\end{array}$} & \multirow{2}{*}{$\begin{array}{l}\text { Spec- } \\
\text { trum }\end{array}$} & \multirow{2}{*}{$\lambda_{\mathrm{air}} \mathrm{A}$} & \multicolumn{2}{|c|}{$\begin{array}{l}\text { Intensity and } \\
\text { character }\end{array}$} & \multirow{2}{*}{$\begin{array}{l}\text { Spec- } \\
\text { trum }\end{array}$} & \multirow{2}{*}{$\lambda_{\text {air }} \mathrm{A}$} & \multicolumn{2}{|c|}{$\begin{array}{l}\text { Intensity and } \\
\text { character }\end{array}$} & \multirow{2}{*}{$\begin{array}{l}\text { Spec- } \\
\text { trum }\end{array}$} \\
\hline & Arc & Spark & & & Arc & Spark & & & Arc & Spark & \\
\hline 5134.05 & 20 & 2 & I & $6365.90^{\mathrm{h}}$ & $25 d$ & & I & 8082.36 & 2 & & I \\
\hline 5135.09 & 200 & 20 & & 6441.14 & $40 c$ & & I & 8178. 16 & $40 c$ & & I \\
\hline 5161.06 & $4 B l$ & & $\mathrm{LuO}$ & 6444.89 & 5 & 15 & II & 8248.68 & 2 & & I \\
\hline 5170.11 & $10 \mathrm{Bl}$ & 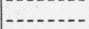 & $\mathrm{LuO}$ & $6463.12^{\mathrm{i}}$ & $400 c$ & 800 & II & 8329.45 & 5 & & I \\
\hline 5196.61 & $10+p$ & $\ldots$ & I & 6477.67 & 30 & 2 & I & 8382.08 & 30 & - & I \\
\hline 5206.47 & 10 & & I & 6523.18 & $80 c$ & 5 & I & 8408.21 & 8 & & I \\
\hline 5250.71 & $2 p ?$ & & I & $6611.7^{\mathrm{j}}$ & $100 c$ & $150 c$ & II & 8459.19 & 150 & 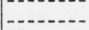 & II \\
\hline 5278.48 & -1 & & II & 6619.15 & 5 & - - & I & 8478.50 & 50 & -1.0. & I \\
\hline 5304.40 & 20 & 2 & I & 6668.74 & $4 c$ & - non & I & 8508.08 & 100 & 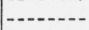 & I \\
\hline 5349.12 & 25 & 2 & I & 6677.14 & 40 & & I & 8610.98 & 120 & - n & I \\
\hline $5402.57^{\mathrm{d}}$ & $150 \mathrm{c}$ & $10 c$ & I & 6735.76 & 5 & & I & 8690.23 & $3 h$ & & I \\
\hline 5421.90 & $50 c$ & 5 & I & 6766.32 & $2 h ?$ & - & I & 8712.78 & 3 & $\ldots$ & II \\
\hline 5437.88 & 30 & 3 & I & 6793.77 & $40 c$ & 2 & I & 8719.00 & $1 e$ & 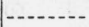 & I \\
\hline 5453.57 & 8 & 1 & I & 6826.59 & 4 & $-\ldots$ & II & 8744.03 & $3 e$ & - & I \\
\hline 5465.50 & $3 ?$ & & I & 6892.55 & 3 & 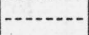 & II & 8788.83 & $4 e$ & - n. & I \\
\hline $5476.69 \mathrm{e}$ & 500 & 1000 & II & 6917.31 & 50 & - & I & 8795.6 & 2 & & I \\
\hline 5505.91 & 1 & 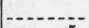 & I & 6943.96 & 5 & $\ldots$ & I & $8949.5^{\text {m }}$ & $40 c$ & & II \\
\hline 5602.56 & 1 & 5 & II & 7031.24 & 50 & $--1-2$ & I & 9116.26 & $20 e$ & - & I \\
\hline 5664.89 & 2 & 20 & II & 7096.34 & $30 c$ & $-0-2-1$ & I & 9203.92 & $8 c$ & - & I \\
\hline 5713.49 & 3 & 15 & II & 7101.98 & $2 ?$ & - n & I & 9273.79 & $2 e$ & - & I \\
\hline 5736.55 & $150 c$ & 15 & I & 7125.84 & 120 & & II & 9282.5 & $1 ?$ & & I \\
\hline 5775.40 & 50 & 5 & I & 7142.79 & 7 & - & I & 9504.50 & $2 e$ & & I \\
\hline 5800.59 & 30 & 2 & I & 7143.10 & 5 & $\ldots$ & I & 9661.69 & $10 e$ & - - - & II \\
\hline 5860.79 & $20 c$ & 2 & I & 7165.94 & 9 & - & II? & 9696.03 & $30 e$ & $-1-2$ & I \\
\hline 5866.30 & 3 & & I & 7196.40 & $5 c$ & $\ldots$ & I & 9841.32 & 2 & -- & II \\
\hline 5887.23 & 1 & $6 h$ & II & 7229.06 & $2 ?$ & & I & 9892.00 & $5 e$ & & I \\
\hline 5961. 39 & $3 c$ & & I & 7237.98 & $40 c$ & & I & 9909.9 & $1 e ?$ & & I \\
\hline $5983.9^{\mathrm{f}}$ & $200 c$ & $400 c$ & II & 7324.97 & $2 ?$ & $\ldots$ & I & 9914.92 & $100 e$ & $\ldots$ & I \\
\hline 5997.13 & 50 & 5 & I & 7409.70 & 7 & $-\ldots$ & II & 10012.46 & $5 c$ & - & I \\
\hline 6004.52 & 400 & 40 & I & 7441.52 & 20 & 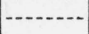 & I & 10114.8 & $2 e$ & $-\cdots$ & I \\
\hline 6041.66 & $20 c$ & 1 & I & 7456.96 & 5 & & II & 10118.54 & $4 e$ & & I \\
\hline 6055.03 & $150 d$ & 10 & I & 7543.06 & 2 & & I & 10282. 26 & $10 e$ & & I \\
\hline 6084.14 & $15 c$ & $\ldots$ & I & 7553.20 & 1 & $\ldots$ & I & 10497.6 & 6 & $\ldots$ & I \\
\hline 6140.71 & $10 c$ & & I & 7640.08 & 9 & - & I & 10680.5 & 8 & . & I \\
\hline 6141.72 & 5 & & I & $7649.54^{\mathrm{k}}$ & $8 c$ & $\ldots$ & I & 10729.8 & 10 & $-\cdots$ & I \\
\hline 6159.94 & 50 & 200 & II & 7659.01 & $4 c$ & & I & 10758. 2 & 5 & & II \\
\hline 6195.12 & $15 c$ & & I & 7686.81 & $2 h$ & & I & 10770.2 & 20 & & I \\
\hline 6199.66 & 40 & 120 & II & 7758.30 & $20 c$ & $\ldots$ & I & & & & \\
\hline $6221.87^{\mathrm{g}}$ & 500 & 1000 & II & 7815.91 & $25 c$ & - & I & & & & \\
\hline 6228.14 & 10 & 40 & II & 7911.62 & $30 c$ & - & I & & & & \\
\hline 6235.36 & 25 & 100 & II & 7912. 32 & $3 ?$ & & I & & & & \\
\hline 6242.34 & 40 & 200 & II & 7917.52 & 15 & $\ldots$ & II & & & 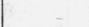 & \\
\hline 6248.80 & $10 c$ & $\ldots$ & I & 7927.58 & $2 ?$ & $-1-2$ & I & & & & \\
\hline 6345.35 & 60 & 4 & I & 7939.63 & 2 & -......... & I & & & & \\
\hline 6354.85 & 20 & 1 & I & 7965.31 & $10 c$ & - & I & & & & \\
\hline
\end{tabular}

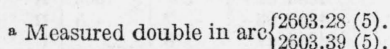

- Nine components according to Schüler and Schmidt [16]

- Thirteen components according to Schüler and Schmidt [16].

d Fifteen components according to Gollnow [17].

- Fifteen components according to Schüler and Schmidt [16].

$f$ Seven components according to Schüler and Schmidt [16].

g Nine components according to Schüler and Schmidt [16].

h Measured double $\{6365.79$ (10).

i Three components according to Schüler and Schmidt [16].

66611.28 (30).

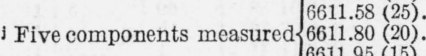
$6611.95(15)$ $6612.04(10)$

k Diffuse double measured $\left\{\begin{array}{l}7649.28(3) \\ 7649.79(3)\end{array}\right.$

1 Unsymmetrical double measured \{7815.84 (15). $8948.93(20)$.

m Three components measured $\left\{\begin{array}{l}8949.63(15) \text {. } \\ 8950.18(10) \text {. }\end{array}\right.$ 


\section{REFERENCES}

[1] B. S. Hopkins, Chemistry of the Rarer Elements (D. C. Heath \& Co., Boston, 1923)

G. v. Hevesy, Die seltenen Erden vom Standpunkte des Atombaues (Julius Springer, Berlin, 1927).

[2] H. Kayser, Handbuch der Spectroscopie 5 and $\boldsymbol{y}$ (S. Hirzel, Leipzig, 1910 and 1934).

[3] A. Hund, Linienspektren und periodisches System der Elemente (Julius Springer, Berlin, 1927).

[4] L. Rolla and G. Piccardi, Phil. Mag. y, 286 (1929).

[5] F. Exner and E. Haschek, Die Spektren der Elemente bei normalem Druck, 2 and 3 (Franz Deuticke, Wien, 1911).

[6] W. F. Meggers and B. F. Scribner, BS J. Research 5, 73 (1930).

[7] G. Urbain, Compt. rend. 145, 795 (1907).

[8] C. Auer v. Welsbach, Sitzber. Akad. Wiss., Wien, 116 [IIb], 1425 (1907).

[9] J. M. Eder and E. Valenta, Sitzber. Akad. Wiss., Wien, 119 [IIa], 3 (1910).

[10] J. M. Eder and E. Valenta, Atlas typischer Spektren (Wien, 1911).

[11] J. M. Eder, Sitzber, Akad. Wiss. Wien 124 [IIa], 707 (1915).

[12] A. S. King, Astrophys. J. y4, 328 (1931).

[13] Eastman Kodak Co., Photographic Plates for Use in Spectroscopy and Astronomy (1935).

[14] Trans. Int. Astron. Union 3, 86 (1928).

[15] K. Burns and F. M. Walters, Jr., Pub. Allegheny Obs. 6, 159 (1929); 8, 27 (1930).

[16] H. Schüler and Th. Schmidt, Z. Physik 95, 265 (1935).

[17] H. Gollnow, Z. Physik 103, 443 (1936).

[18] H. Schüler, and Th. Schmidt, Naturwiss. 22, 714 (1934).

[19] G. Urbain, Compt. rend. 152, 141 (1911).

Washington, May 5, 1937. 